\title{
Smile in a Box
}

The Straumann Smile in a Box is a digital, modular, integrated treatment planning and manufacturing service that caters to various surgical and prosthetic indications. It enables you to outsource parts of the digital workflow as and when required - you simply select the services you desire and Straumann will deliver everything you need in a single box.

This solution simplifies your workflow, saves you time and facilitates business growth while maintaining the highest quality of patient care and expanding the services you offer.

Enjoy all the benefits of digital dentistry without having to invest in the technology yourself.
For more information on the Straumann Smile in a Box, visit www.straumann.com/ digital/en/discover/smile-in-a-box.html

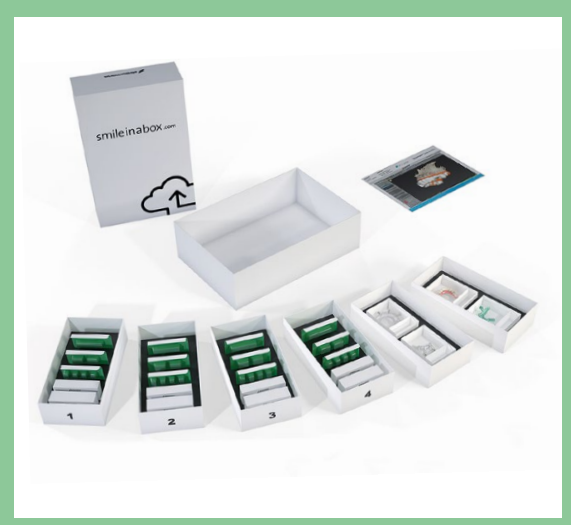

\section{Changes to payroll giving you a headache?}

Efficient payroll keeps every dental practice running smoothly.

With changes to payroll in this tax year and the next, consider outsourcing it to the premium provider, Wagemate.

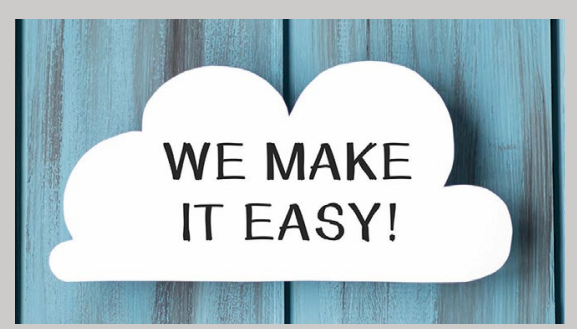

Whether your practice is large or small, with payroll in the hands of specialists, people will be paid accurately, on time and you will be fully compliant with the latest legislation.

Wagemate will manage everything, leaving you free to focus on growing your business, knowing payroll is taken care of. We also use the latest technology to ensure quality.

To better manage your payroll contact Wagemate on 03330102102 or via email at info@wagemate.com.

\section{Remember the colour code}

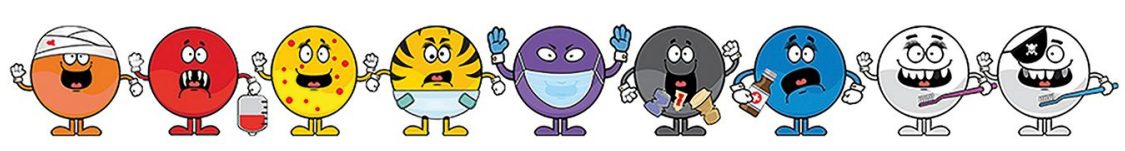

Does your team sometimes find it hard to remember which waste belongs where?

Help ensure they never make a mistake during waste disposal with Colour Code Character Posters from Initial Medical.

Personifications of each waste stream, these fun, vibrant characters make learning the Department of Health's colour coded guidelines for best practice waste disposal easy and stress-free. By attaching visual aids as well as the colour to each waste stream, people are likely to remember the

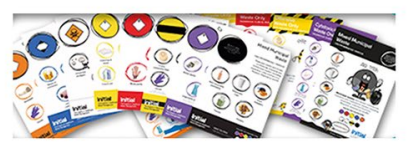

correct disposal method for each type of waste and what items are classified under each umbrella.

For further information and to download the free poster visit www.initial.co.uk/ medical, www.initial.co.uk/colour-codingguide/ or call 08708504045 .

\section{Next gen announcements}

Align Technology, Inc. (NASDAQ:

ALGN) has announced the availability of the iTero Element Plus Series, which expands the company's portfolio of iTero Element scanners and imaging systems to include new solutions that serve a broader range of the dental market.

The new iTero Element Plus Series of scanners and imaging systems builds on the success of the award-winning iTero Element family and offers all of the existing orthodontic and restorative digital capabilities doctors have come to rely on - plus faster processing time and advanced visualisation capabilities for a seamless scanning experience in a new sleek, ergonomically designed package.

Available in both cart and mobile configurations, the iTero Element Plus Series offers increased flexibility and mobility. The mobile configuration makes the power of the iTero Element Plus Series portable with a medical grade, compact mobile scanner solution that delivers the same high-quality images as the cart configuration.

The iTero Element Plus Series of solutions offer restorative and orthodontic digital workflows with:

$\rightarrow$ Enhanced visualisation for optimised patient experience, with higher brightness for clearer and more vivid images, and a fully integrated $3 \mathrm{D}$ intraoral camera

$\rightarrow$ Faster, seamless scanning: 20 percent less waiting time for scans to process

$\rightarrow$ Future AI-based features

$\rightarrow$ The iTero Element 5D Plus imaging system includes NIRI technology, which aids in the detection of interproximal caries. The iTero Element 5D Plus Lite imaging system allows for easy NIRI activation via a software upgrade.

Information about the iTero Element Plus Series can be found at http://www. itero.com

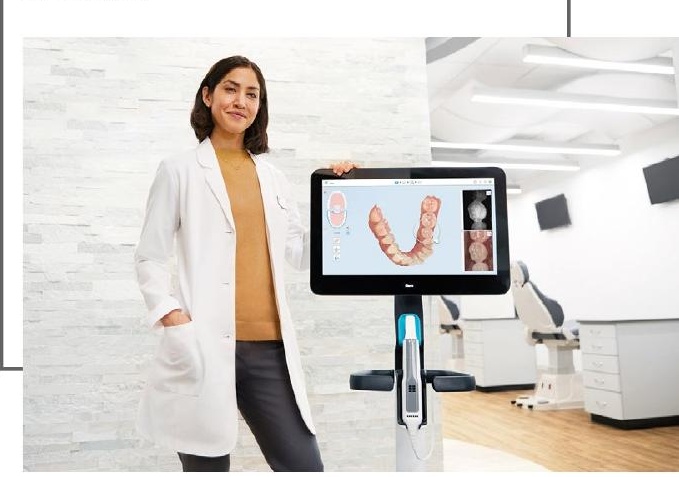

